\title{
LOCALIZATION OF WINDOWS AND DOORS IN 3D POINT CLOUDS OF FACADES
}

\author{
William Nguatem, Martin Drauschke, Helmut Mayer \\ Institute of Applied Computer Science \\ Bundeswehr University Munich \\ Werner-Heisenberg-Weg 39, 85577 Neubiberg, Germany \\ william.nguatem, martin.drauschke, helmut .mayer@unibw.de \\ http://www . unibw.de/inf4/professuren/photo/index_html
}

Commission III/2,4

KEY WORDS: Point Cloud, Object Localization, Splines, Model Selection, Non-linear Regression Fitting, Markov Chain MonteCarlo

\begin{abstract}
:
In this paper, we present a fully automatic approach to localize the outlines of facade objects (windows and doors) in 3D point clouds of facades. We introduce an approach to search for the main facade wall and locate the facade objects within a probabilistic framework. Our search routine is based on Monte Carlo Simulation (MC-Simulation). Templates containing control points of curves are used to approximate the possible shapes of windows and doors. These are interpolated using parametric B-spline curves. These templates are scored in a sliding window style over the entire facade using a likelihood function in a probabilistic matching procedure. This produces many competing results for which a two layered model selection based on Bayes factor is applied. A major thrust in our work is the introduction of a 2D shape-space of similar shapes under affine transform in this architectural scene. This transforms the initial parametric B-splines curves representing the outlines of objects to curves of affine similarity in a strongly reduced dimensionality thus facilitating the generation of competing hypotheses within the search space. A further computational speedup is achieved through the clustering of the search space to disjoint regions, thus enabling a parallel implementation. We obtain state-of-the results on self-acquired data sets. The robustness of our algorithm is evaluated on 3D point clouds from image matching and LiDAR data of diverse quality.
\end{abstract}

\section{INTRODUCTION}

The modeling of architectural scenes is a very busy research topic and can be viewed from very different perspectives: The nature of the data (images vs. LiDAR, aerial vs. terrestrial), its scale (building vs. city), or its purpose, e.g., cultural heritage, construction planning or touristic applications. Consequently, the employed methodologies also differ. For examples, Lin et al. (2013) concentrate on fast data processing methods to reconstruct suburban areas from a mobile mapping system using LiDAR, Lafarge and Mallet (2012) derive a scene representation at city-scale in which simple surfaces are modeled by geometric primitives, and complex structures are represented by a triangular mesh. While Friedman and Stamos (2013) focus on repetitive structures of modern buildings, Brandenburger et al. (2013) extract ornamental details from images of historical facades.

Since man-made objects like buildings show a rich diversity in size, shape, or style, all approaches for reconstructing, approximating or interpreting such data have to deal with model selection, i.e., methods for fitting and comparing competing models to the acquired data are employed. If scenes are only coarsely modeled, the presentation of the approaches often concentrate on data representation, e.g., (Lafarge and Mallet, 2012), or the focus of the publication lies on the modeling of domain knowledge or its transfer during the data analysis, e.g., (Becker and Haala, 2008; Friedman and Stamos, 2013; Lin et al., 2013). The model selection is often only sketched. In contrast, when modeling smaller structures, often several models roughly fit to the data. Then in this case model selection methods are often discussed to demonstrate the plausibility of the model refinements, e.g., (Alegre and Dellaert, 2004; Dick et al., 2004; Brandenburger et al., 2013).

Which model fits best to the data? This is a very old and philosophical question. Already in the 14th century, William of Ock- ham stated that it is better to choose a simple model than a complex one. This principle is known as Occam's razor. Since this judgement is sensible, many modern model selection approaches in computer sciences consider both, the closeness of the model to the data, and the model complexity. E.g., Akaike (1973) derived his selection criterion from information theory, and Rissanen (1987) proposed the minimum description length (MDL) principle for choosing the best model, i.e., he evaluates its size for representation.

A probabilistic view on model selection is proposed by Alegre and Dellaert (2004) who interpret a rectified facade image by its stepwise division in meaningful parts of rectangular shape. The segmented parts are interpreted by a Bayesian generative model which was constructed from a context-free grammar. Markov Chain Monte Carlo (MCMC) sampling is employed to derive posterior probabilities for each part. Another probabilistic Bayesian framework is proposed by Dick et al. (2004) who use one complex model for windows of several shapes, e.g., with arch height and bevels on the one hand, and various priors, e.g., on object shape and position on the other hand. The evaluation is based on likelihoods derived from image intensities of backprojected facade objects. Besides the high complexity of the MCMC-based search for facade objects, the approach has another deficit regarding the assumption on normalized illumination condition and a uniform material characteristic of each object. In probabilistic approaches, model complexity is considered when defining likelihood terms, e.g., (Dick et al., 2004), or in the probabilities for jumps of the reversible jump Markov Chain Monte Carlo (rjMCMC) framework, e.g., (Ripperda and Brenner, 2006).

In photogrammetric research, several approaches achieve satisfying results for detecting rectangular doors and windows. For instance Becker and Haala (2008) derive building models with windows and doors from LiDAR point clouds by detecting 3D 
edge points in the point cloud, and an axis-aligned cell decomposition to obtain outlines of doors and windows. The authors state that arch-shaped doors can also be considered, but the corresponding model selection step is not discussed in (Becker and Haala, 2008). We assume that the classification is performed by a decision stump, hence arches with a small height are not detected. Recently, Fritsch et al. (2013) adopted the approach to dense point clouds derived from 3D reconstruction. Another approach for building analysis in LiDAR point clouds is presented by Schmittwilken et al. (2009) who derive the scene interpretation by employing a conditional random field and an attribute grammar to interpret geometrical entities which were recognized before. While this approach combines a probabilistic and a flexible model-based interpretation, the shape of the recognized building parts only relies on the previous segmentation step which is somehow similar to (Becker and Haala, 2008). As third example, Tuttas and Stilla (2013) derive building models from oblique ALS, and they derive window hypotheses from $3 \mathrm{D}$ points reflected by objects within the rooms (voyeur effect) assuming glass windows. Only rectangular shaped windows are presented in their results, although the facade shows arch-shaped windows.

All the mentioned state of the art approaches have one step in common: First a coarse building model is determined, i.e., the major planes of ground, facade and roof, and then, it is refined in a successive step. Yet, in our experiments we saw that it is sometimes difficult or even impossible to find the perfect facade plane, because of a highly structured facade in $3 \mathrm{D}$ due to ornaments, balconies and oriels, or because of the building construction, especially considering historical buildings and old buildings in rural areas. Consequently, state of the art approaches may have problems when detecting building parts in such environments. We want to overcome this drawback by considering a discrete set of segmented planes for facade hypothesis rather than a single perfect segmentation of the facade.

In this paper we propose a window and door detection based on sampling with only few iterations, which works well on various kinds of facades with a geometric structure in 3D, and as demonstrated in our experiments we are able to reliably distinguish between various window and door shapes. Specifically, we want to estimate a precise outline of all windows of a facade, which has been reconstructed from image sets by recent approaches, e.g., (Snavely et al., 2006; Frahm et al., 2010; Mayer et al., 2012). We use MC-Simulation to generate and score competing models and apply a model selection based on Bayes factor. For the parametrization of the models, we use the notion of sub-space from (Isard and Blake, 1998) which originally has been proposed in the field of object contour tracking.

The rest of the paper is structured as follows; In Section 2, beginning with an introduction, we present our probabilistic facade object localization algorithm in its entirety. This is followed by a thorough discussion of our results in the evaluation presented in Section 3, and finally, in Section 4, we conclude and present possible future work.

\section{PROBABILISTIC OBJECT LOCALIZATION ON FACADES}

\subsection{Overview of the Algorithm}

The input to our system is an unstructured $3 \mathrm{D}$ point cloud, $\mathcal{D}$, containing one or more building facades. This could have originated from image matching or LiDAR. The output are fitted contours defining the outlines of windows and doors. We divide the work flow of our algorithms into three main stages: Facade Segmentation, Window and Door Localization, and Model Selection. Within the Facade Segmentation, assuming that $\mathcal{D}$ underlies a known metric scale and the gravity (up) vector $\mathbf{v}$ is known, we extract facades planes and estimate the outlines of interesting objects present on the facade. In the localization stage, making no assumptions about the occurrences of windows and doors on facades, we build probability distributions of these objects on every location of the facade by matching predefined templates on the estimated outlines. This results in multiple detections of competing templates. We remedy this during the model selection stage. Figure 1 depicts the detailed work flow of our algorithm.

\subsection{Facade Segmentation}

There exist a plethora of work describing oriented plane segmentation in 3D point clouds. However, in the real world, a single segmented vertical plane from the 3D point clouds of a facade is hardly enough to capture information about the true boundaries of facade objects e.g. windows. Reasons are for instance architectural imperfections, variability in shapes and styles of windows and doors as well as variability in the distances these off-thefacade objects protrude from the true facade. Also, a possible unequal point distribution on the facade originating from textural differences could possibly be an additional problem. To remedy this we first segment a single vertical plane using MSAC from (Torr and Zisserman, 2000). This serves as a starting hypothesis for the construction of a discrete probability distribution over facades. We sweep this first segmented plane along its normal direction, whilst randomly changing the angle of the vertical direction (the value of $\mathbf{v}$ ) at each sweeping step. We call this procedure a plane-angular sweep. The small and random changes in $\mathbf{v}$ during plane sweeping ensures the construction of an ensemble of planes slightly but non-parallel to the first main segmented plane. For the plane-angular sweeping, a finer inlier threshold is used compared to that used within MSAC to segment the first plane. For $\mathcal{M}$ such plane-angular sweeps, the output for a given facade are a finite set of $\mathcal{M}$ segmented planes, $\left\{\mathcal{F}^{(i)}\right\}_{i=1}^{\mathcal{M}}$, used as a discrete representation of $p(\mathcal{F} \mid \mathcal{D})$, the probability of the true facade given the data. For each element of $\left\{\mathcal{F}^{(i)}\right\}_{i=1}^{\mathcal{M}}$, we estimate boundary points using a boundary estimation algorithm e.g. (Rusu et al., 2007). These boundary points $\left\{\mathcal{B}^{(i)}\right\}_{i=1}^{\mathcal{M}}$, are a discrete approximation of the probability of the outlines of facade objects. Figure 2 shows on the left side the inliers of two planeangular sweeps of a reconstructed facade points and the right side depicts hypothesis of the estimated object boundaries. Taking the windows on this facade as an example, a single facade hypothesis from plane segmentation makes only a partial coverage of the presence and locations of these facade objects. In some simple cases, a single sweep's boundary hypothesis can reveal almost all the outlines of windows and doors. This is the case shown in 3 for these facades gained from $3 \mathrm{D}$ point clouds from image matching.

Optionally, for buildings whose windows and doors protrude very little from the facade, surface curvature of $3 \mathrm{D}$ points are estimated using e.g. algorithms presented in (Nguatem et al., 2012). Points exhibiting high curvature values are then fused to every element of $\left\{\mathcal{B}^{(i)}\right\}_{i=1}^{\mathcal{M}}$.

\subsection{Window and Door Localization}

The goal of the detection stage is to localize portions of $\left\{\mathcal{B}^{(i)}\right\}_{i=1}^{\mathcal{M}}$ with high probability of being the outlines of windows and doors. Using orthogonal projection, we convert these boundary estimates from $3 \mathrm{D}$ to $2 \mathrm{D}$ points, $\left\{\mathcal{B}_{2 D}^{(i)}\right\}_{i=1}^{\mathcal{M}}$. On every element of $\left\{\mathcal{B}_{2 D}^{(i)}\right\}_{i=1}^{\mathcal{M}}$, using MC-Simulation in a sliding window style, we 


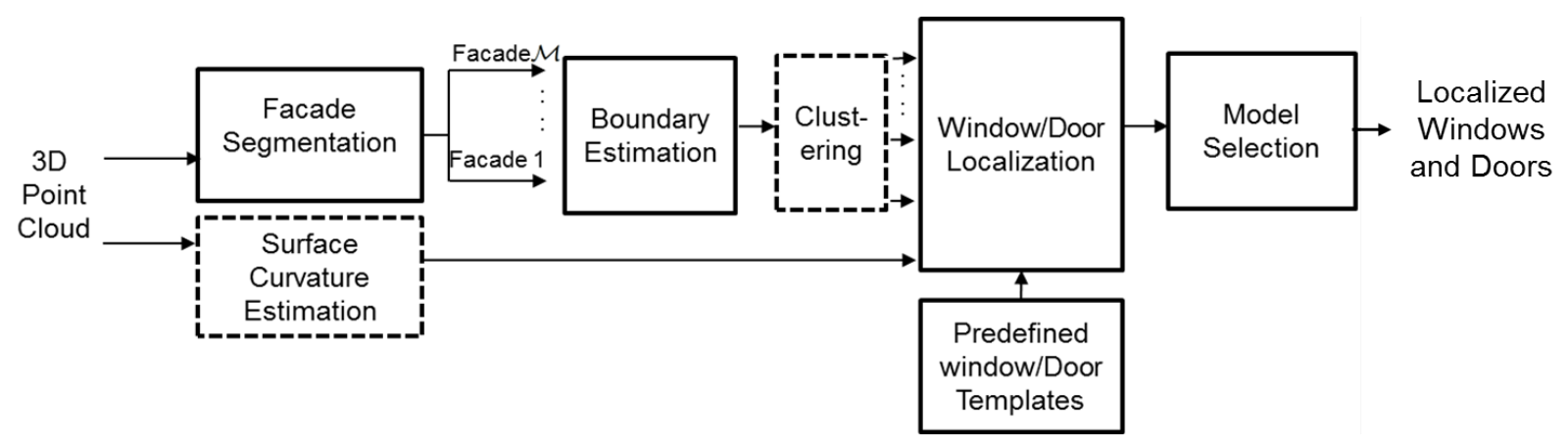

Figure 1: Block diagram of the complete algorithmic work flow. An optional clustering is used to divide the search space to enable a parallel implementation. Also the fusion of surface curvature values is optional and only needed for facades whereby, windows and doors are only slightly off the main facade plane thus providing little 3D geometrical information. This can be found particularly in some modern buildings.

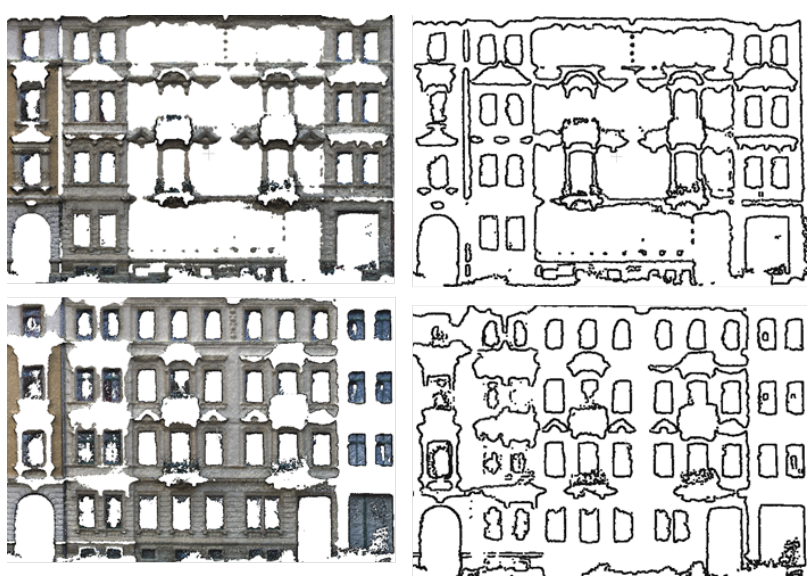

Figure 2: The left side shows two hypothesis from plane-angular sweep segmentation of the same facade and the corresponding outlines are shown on the right. These combined outlines in most cases reveals the correct outlines of windows and doors.

search and score predefined templates for windows and doors using a window and door likelihood function. In Figure 3, we can see that huge portions of the boundary estimates contain no 3D point. Optionally, to avoid sliding over these big empty regions, we cluster $\left\{\mathcal{B}_{2 D}^{(i)}\right\}_{i=1}^{\mathcal{M}}$ and apply the sliding window only on clusters of reasonable sizes. For very long facades, this results in a significant speedup.

2.3.1 Notation, Model Definition and Parametrisation Before explaining our probabilistic template matching algorithm, we first define the notations used and the choice of parametrisation for window and door templates. Figure 4 illustrates the span of windows and door models used during this research and the corresponding templates. Analogous to approaches on shape analysis, e.g., Ferrari et al. (2006) and Riemenschneider et al. (2010), we define these templates by control points (the blue stared markers) whose 2D coordinates when interpolated gives the full outlines (the red curves) of the model $\mathbf{r}(s)$. This interpolation is performed using parametric B-spline curves and is defined as follows

$$
\mathbf{r}(s)=\left(\begin{array}{cc}
\mathbf{B}(s)^{T} & 0 \\
0 & \mathbf{B}(s)^{T}
\end{array}\right) \mathbf{Q}
$$

where $\mathbf{Q}=\left(x_{1}, x_{2}, x_{3}, \ldots, x_{N}, y_{1}, y_{2}, y_{3}, \ldots, y_{N}\right)^{T}$ defines the coordinates of the control points, $\mathbf{B}(s)$ a spline basis function and $N$ is the number of control points. The choice of interpola-
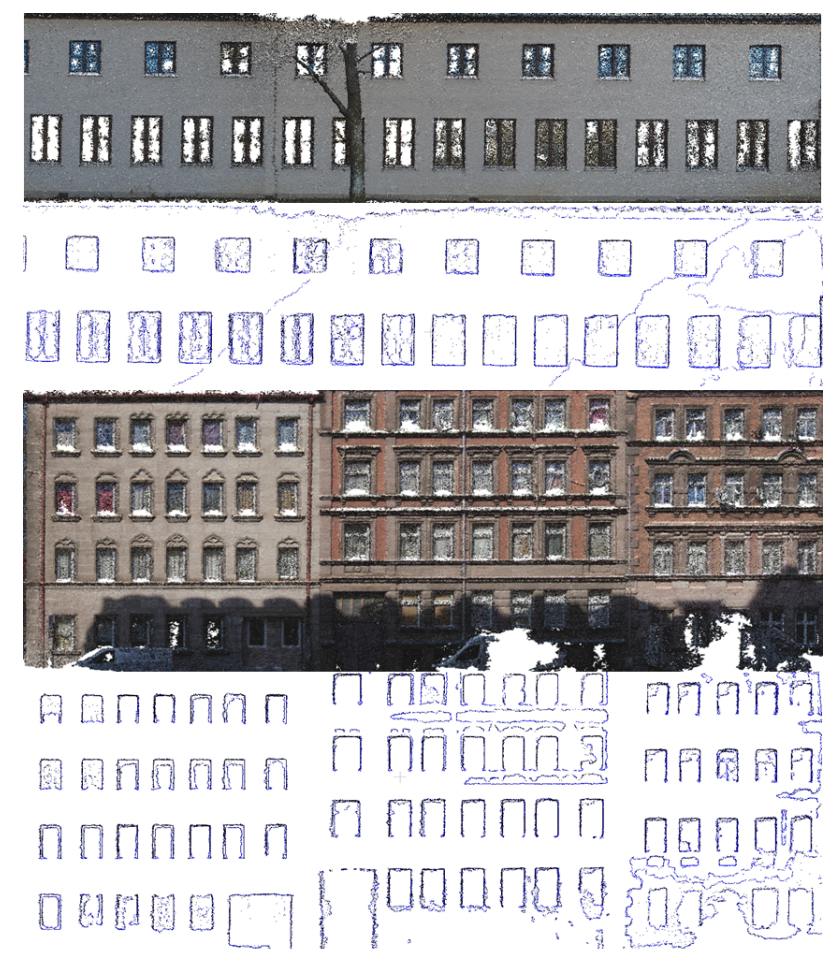

Figure 3: The point clouds of two facades are shown above a single hypothesis boundary estimates. The boundary estimates reveals the outlines of most windows and doors present on the facades.

tion using the B-spline representation brings along some advantages: First, this representation enforces the natural smoothness inherent in the object outlines. Secondly, this turns out to be more robust to measurement noise than explicit parametrisation, and it reduces the dimensionality considerably. We further reduce the dimensionality of these templates by defining a mapping from the spline space of control points to a much lower dimensional sub-space, the 2D affine shape-space, with a shape-space vector, $\boldsymbol{\theta}$, given by

$$
\mathbf{Q}=\mathbf{W} \boldsymbol{\theta}+\mathbf{Q}_{\mathbf{0}}
$$

where $\mathbf{Q}_{\mathbf{0}}$ defines the template, and $\mathbf{W}$ the shape-space matrix. $\mathbf{W}$ is defined as

$$
\mathbf{W}=\left[\begin{array}{cccc}
1 & 0 & \mathbf{Q}_{0}^{\mathrm{x}} & \mathbf{0} \\
\mathbf{0} & 1 & 0 & \mathbf{Q}_{0}^{\mathrm{y}}
\end{array}\right]
$$



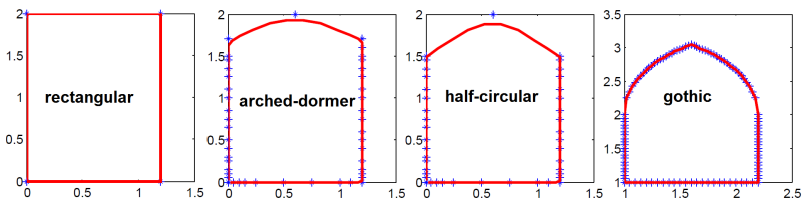

Figure 4: The span of windows and doors templates, $\mathbf{Q}_{\mathbf{0}}$, used during this work. This ranges from the simple rectangular represented by the four corner points (marked blue) through the arched-dormer, half-circular and gothic windows and doors represented by many more control points.

where $\mathbf{0}=(0,0, \ldots, 0)^{T}$ and $\mathbf{1}=(1,1, \ldots, 1)^{T} \cdot \mathbf{Q}_{\mathbf{0}}^{\mathbf{x}}$ and $\mathbf{Q}_{\mathbf{0}}^{\mathbf{y}}$ are the decomposition of the template curve $\mathbf{Q}_{0}$ into vectors of its $x$ and $y$ coordinates respectfully. Thus mapping in equation (2) can be written as follows

$$
\left[\begin{array}{l}
\mathbf{Q}^{\mathbf{x}} \\
\mathbf{Q}^{\mathbf{y}}
\end{array}\right]=\mathbf{W} \boldsymbol{\theta}+\left[\begin{array}{l}
\mathbf{Q}_{0}^{\mathbf{x}} \\
\mathbf{Q}_{0}^{\mathbf{y}}
\end{array}\right]
$$

Elements of $\boldsymbol{\theta}$ are given by

$$
\boldsymbol{\theta}=\left(t_{x}, t_{y}, s_{x}, s_{y}\right)^{T}, \text { where }
$$

$t_{x}=$ a shift in the $\mathrm{x}$-axis $\quad t_{y}=\mathrm{a}$ shift in the $\mathrm{y}$-axis

$s_{x}=$ scaling in the $\mathrm{x}$-axis $s_{y}=$ scaling in the $\mathrm{y}$-axis

Examples of 2D shape-space vectors and the respective meaning of the affine transformations on the template are:

- $\boldsymbol{\theta}=(0,0,0,0)^{T}$, the original template $\mathbf{Q}_{\mathbf{0}}$

- $\boldsymbol{\theta}=(0,1,0,0)^{T}, \mathbf{Q}_{\mathbf{0}}$ shifted 1 unit to the left

- $\boldsymbol{\theta}=(0,0,1,1)^{T}, \mathbf{Q}_{\mathbf{0}}$ doubled in size.

This mapping ensures that changes in the components of $\boldsymbol{\theta}$ results in all the necessary affine transformations of similarities by our templates. We neglect a rotation term since windows and doors are assumed to be placed up-right on the facade, knowing that our vertical up direction is reliable. If $\boldsymbol{\theta}$ is defined over a compact support $\mathcal{S}$, then for a given template from the span, the only elements of $\boldsymbol{\theta}$ which are expected to vary are $s_{x}$ and $s_{y}$ to enable the capturing of the different windows and door sizes and $t_{x}$ and $t_{y}$ for shifting the templates to different positions of the facade. However, the later is implicitly gained through the sliding of the template over the facade and is deterministic. This leaves an effective dimensionality of two and therefore substantiates the choice of shape-space over the spline space parametrisation.

2.3.2 Template Likelihood Computation In this section we derive a measure of how good a realization of $\boldsymbol{\theta}$ from $\mathcal{S}$ explains the data. Ideally, we would like a template, when placed on a particular position on an element of $\left\{\mathcal{B}_{2 D}^{(i)}\right\}_{i=1}^{\mathcal{M}}$ to exhibit perfect fit, i.e. all the inliers should lie uniformly spread on the interpolated curve. MSAC is usually used to capture the goodness of the fit. A good approximation of the point spread is the standard deviation $\sigma(\boldsymbol{\theta})$ of the number of inliers along the curve. This value is inversely proportional to the goodness of the inliers point spread. Uniformly spread inliers have a lower standard deviation than non-uniformly spread points. These two terms, normalized between 0 and 1 are combined to define our window or door outline likelihood function as follows

$$
\mathcal{L}(\hat{\boldsymbol{\theta}}) \propto w(\boldsymbol{\theta})+\sigma(\boldsymbol{\theta}),
$$

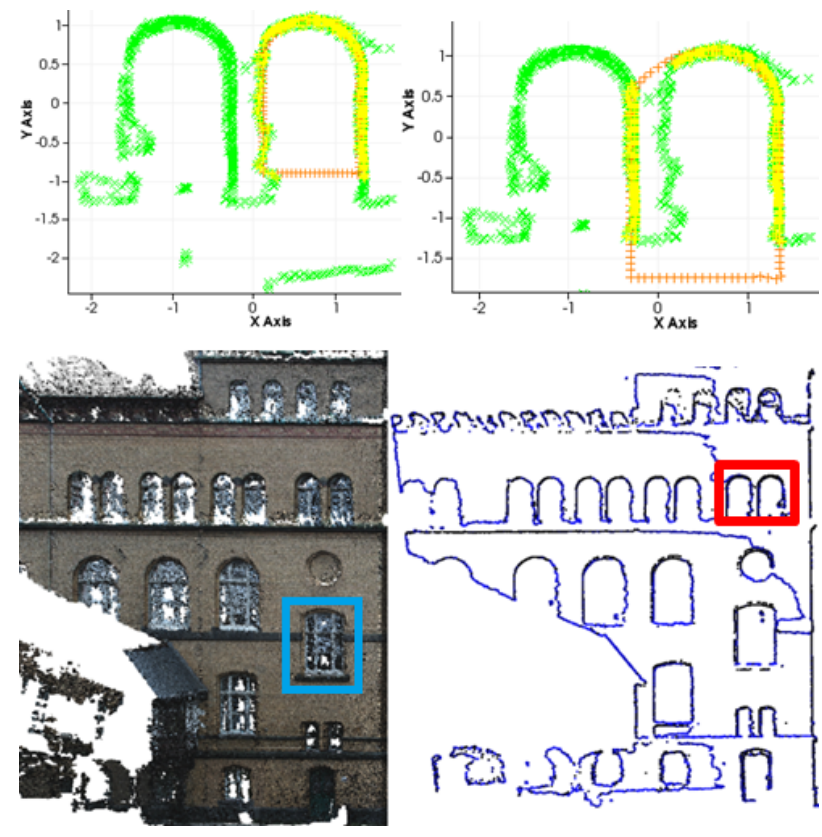

Figure 5: The bottom row shows on the left hand side the input $3 D$ point clouds and the right hand a single element of $\left\{\mathcal{B}_{2 D}^{(i)}\right\}_{i=1}^{\mathcal{M}}$. An enlarged portion of the red marked area is shown on the top row. The green crosses are the boundary points, the brown pluses are 100 interpolation points of the B-spline curve used in fitting the data. The inliers of these curves are shown by the yellow crosses.

where

$$
w(\boldsymbol{\theta}) \propto \exp \left(-\sum_{k} \rho\left(e_{k}^{2}\right)\right),
$$

$\rho$ is

$$
\rho\left(e_{k}^{2}\right)= \begin{cases}e_{k}^{2} & e_{k}^{2}<T^{2} \\ T^{2} & e_{k}^{2} \geq T^{2}\end{cases}
$$

$e_{k}^{2}$ is the shortest distance from the $2 \mathrm{D}$ point $\mathbf{p}_{\mathbf{k}}$ to the interpolated template curve defined by $\boldsymbol{\theta}$ and $T$ is the inlier threshold. The summation index $k$ runs over all the points in the element of $\left\{\mathcal{B}_{2 D}^{(i)}\right\}_{i=1}^{\mathcal{M}}$ considered. In Figure 5, the green crosses are the boundary points and the brown pluses represents 100 points on the interpolated curve for the template of a half-circular window. It may seem that the interpolated curve on the left side of the top row of the Figure has a better fit to the data than the one on the right side (the yellow crosses represent the inliers). Yet, the inliers on the curve of the right hand side show a better average spread (lower $\sigma(\boldsymbol{\theta})$ ) than the ones on the right. This results in an overall reduction in the likelihood of the curve on the right to that on the left and substantiates our choice of likelihood function over the vanilla MSAC.

2.3.3 Probabilistic Template Matching We define the goal of the probabilistic template matching as follows: Given a compact support $\mathcal{S}$ over $\boldsymbol{\theta}$, and a span of templates, compute the likelihood $\left(\mathcal{L}(\boldsymbol{\theta})_{1}, \mathcal{L}(\boldsymbol{\theta})_{2}, \mathcal{L}(\boldsymbol{\theta})_{3}, \ldots \mathcal{L}(\boldsymbol{\theta})_{\mathcal{N}}\right)$ of $\mathcal{N}$ realisations of $\boldsymbol{\theta}$ for every position of each element of $\left\{\mathcal{B}_{2 D}^{(i)}\right\}_{i=1}^{\mathcal{M}}$ and find the maximum. We defined this optimization as follows

$$
\hat{\boldsymbol{\theta}}=\underset{\boldsymbol{\theta} \in \mathcal{S}}{\operatorname{argmax}}(\mathcal{L}(\boldsymbol{\theta}))
$$

For each template $\mathbf{Q}_{0}$ from the span, we solve this optimization using MC-Simulation in the algorithm depicted in Algorithm (1).

Table 1 defines three main categories of variations of windows 


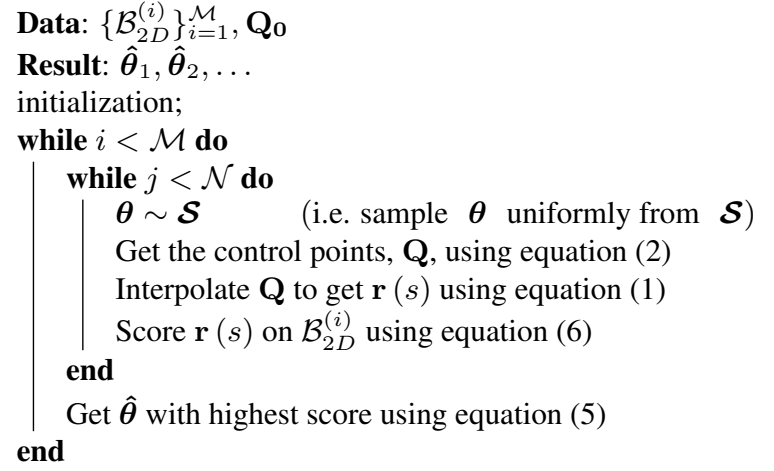

Algorithm 1: Probabilistic template matching.

\begin{tabular}{lccr} 
Categories & Type- 1 & Type-2 & Type-3 \\
\hline$h_{\text {door }}$ & $0.8 \mathrm{~m}-1.3 \mathrm{~m}$ & $1.7 \mathrm{~m}-2.3 \mathrm{~m}$ & $1.3 \mathrm{~m}-1.7 \mathrm{~m}$ \\
$w_{\text {door }}$ & $0.8 \mathrm{~m}-1.4 \mathrm{~m}$ & $1.4 \mathrm{~m}-2.3 \mathrm{~m}$ & $2.3 \mathrm{~m}-3.0 \mathrm{~m}$ \\
$h_{\text {window }}$ & $1.8 \mathrm{~m}-2.6 \mathrm{~m}$ & $2.6 \mathrm{~m}-3.3 \mathrm{~m}$ & $3.3 \mathrm{~m}-3.5 \mathrm{~m}$ \\
$w_{\text {window }}$ & $0.8 \mathrm{~m}-1.4 \mathrm{~m}$ & $1.4 \mathrm{~m}-2.0 \mathrm{~m}$ & $2.0 \mathrm{~m}-3.5 \mathrm{~m}$
\end{tabular}

Table 1: Variations of window and door heights and widths of our experiments. These define a compact support $\mathcal{S}$ over $\boldsymbol{\theta}$.

and doors heights $\left(h_{d}\right)$ and widths $\left(w_{d}\right)$ respectively and this intend defines the compact support $\mathcal{S}$ over $\boldsymbol{\theta}$.

A snapshot of the results of this probabilistic template matching for the input data from Figure 5 is shown in Figure 6 for two different templates. This reveals the problem of multiple detections. Our approach to solving this problem is presented in the next section.

\subsection{Model Selection}

For the rectangular template, linear parametric B-spline curves (also known as a spline of degree one) are used for interpolation. This is equivalent to connecting the four corner points of the rectangle shown in Figure 4. For the other templates, quadratic Bspline curves (splines of degree two) are employed to produce smooth interpolated curves. Though most popular model selection approaches, e.g. AIC, explicitly need to include this difference in dimensionality, we argue that the Bayes factor implicitly penalizes complex models compared to simpler ones when samples are generated from independent and identically distributed (i.i.d) random variables.

The problem of multiple detections can be divided into two main groups: Overlap detection of different templates and overlap detections of the same template but sampled from different categories of the compact support. In a model selection sense, these are all competing detections. We apply a two layered solution called inter class model selection and intra class model selection, respectively.

2.4.1 Intra Class Model Selection Here, all models originate from one and the same template from the span and the ultimate goal is to select one of the category (Type-1, Type-2, Type-3) from Table 1. Let us consider the case where we are using a rectangular template. Using only two samples, i.e., $\mathcal{N}=2$, for each category of the compact support, Figure 7 shows screen shots of the output for a given run of our algorithm at a given position while sliding over an element of $\left\{\mathcal{B}_{2 D}^{(i)}\right\}_{i=1}^{\mathcal{M}}$. The yellow points represent the inliers and the corresponding sampled rectangular curve (brown pluses) depicts a window hypothesis. It can be seen that the two rectangles on the lower row fit this data better than
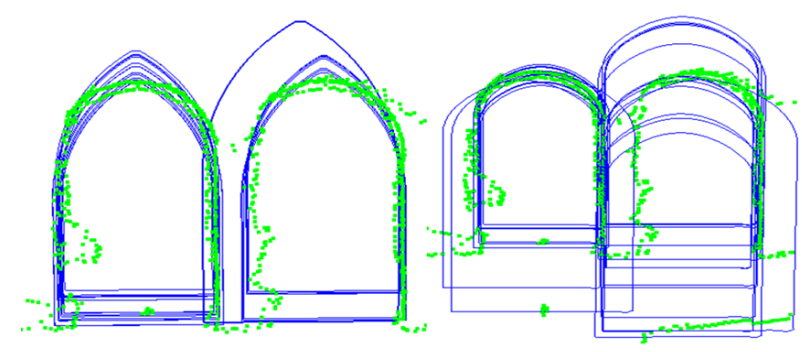

Figure 6: Multiple detections of a half-circular and a gothic window models.
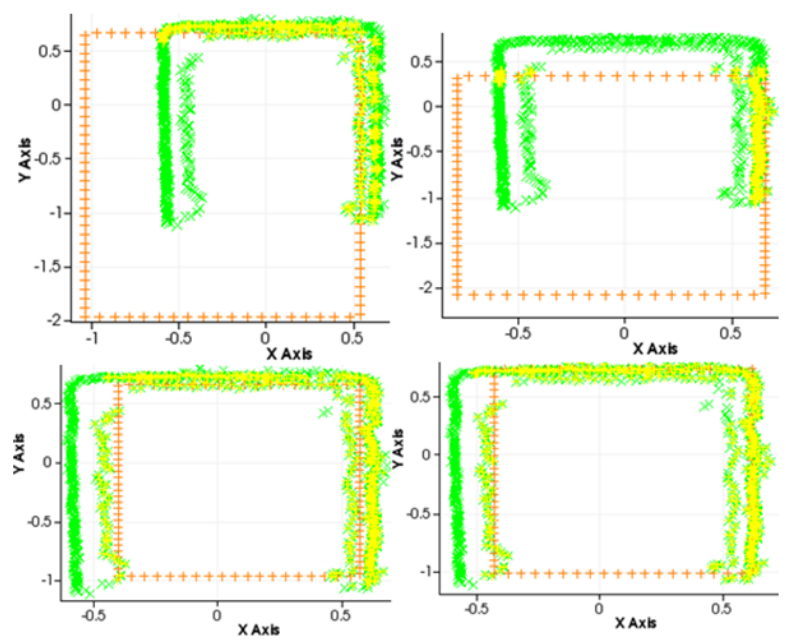

Figure 7: Intra class model selection using the simple rectangular template. The upper row depicts two samples (the brown pluses) of a rectangular template of Type-2 from Table 1 while the lower row shows two samples of Type-1. The strong evidence of Type-1 rectangular window over Type-2 can be seen by the higher number of inliers (yellow crosses) on the samples of the lower row.

the two on the upper row. Since the likelihood function captures the goodness of this fit, one of these two lower rectangles would have the highest score for $\mathcal{L}(\boldsymbol{\theta})$. We call this the maximum a posteriori sample (MAP), $\boldsymbol{\theta}_{\text {rect }}$ for the rectangular template. In accordance to Bayes factor, we select the category from where $\boldsymbol{\theta}_{\text {rect }}$ was sampled (Type-1) in this case.

2.4.2 Inter Class Model Selection Having solved the problem of intra class model selection on all sliding positions as explained above, over the entire facade MAP values are available for all templates from the span, i.e. $\boldsymbol{\theta}_{\text {gothic }}, \boldsymbol{\theta}_{\text {arc }}, \boldsymbol{\theta}_{\text {circle. Now, }}$ the ultimate goal is to choose one of the four competing templates. Again, in accordance with Bayes factor, at a given position on the facade we chose the template whose MAP value at that position is highest. Finally, we select all non-overlapping curves with the highest MAP values. Example MAP estimates of an arched-dormer and a gothic window are compared in Figure 8 demonstrating our inter class model selection.

Since the no assumption about the sizes and placement of windows and doors is made, the end results are estimates of windows and doors from i.i.d samples. This can be seen on the examples in Figures 9, 12 and 10 in the form of different sizes of the localized object outlines, filled in red giving $3 \mathrm{D}$ polygons. We can however, use this assumption and vote between any two neighbouring windows or doors for the most probable size. Occasionally, there will be false localizations as shown in Figure 11. 

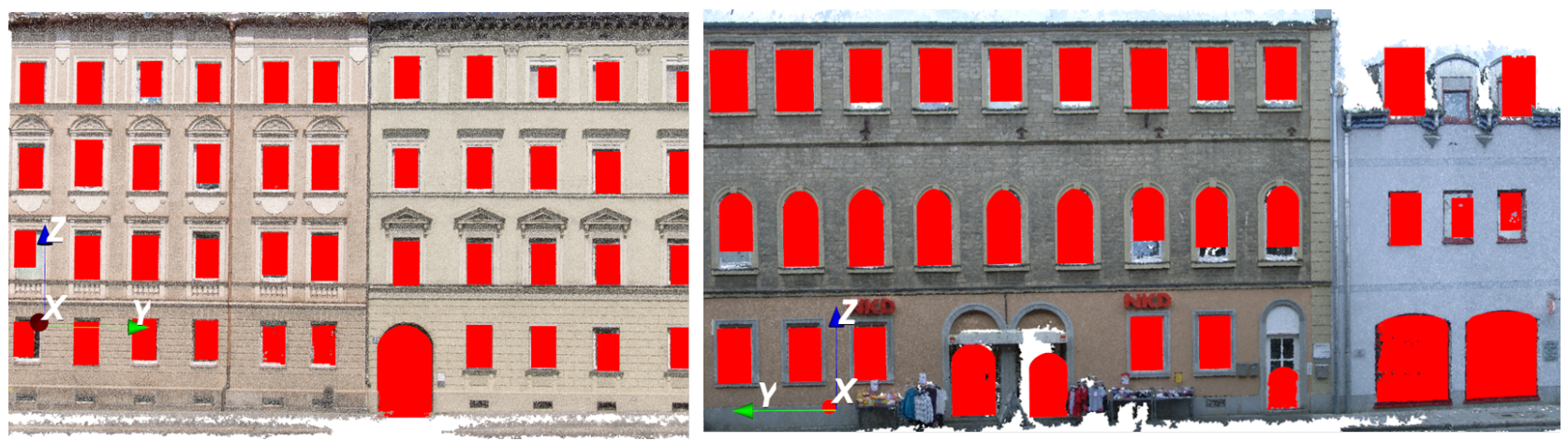

Figure 9: The diagram shows localization results of two different facades. The outlines filled in red giving $3 D$ polygons represent windows and doors. The dimensions of window and door estimates may not be the same. This is because these estimates are from i.i.d random variables. This can be seen on three of the doors on the right facade.

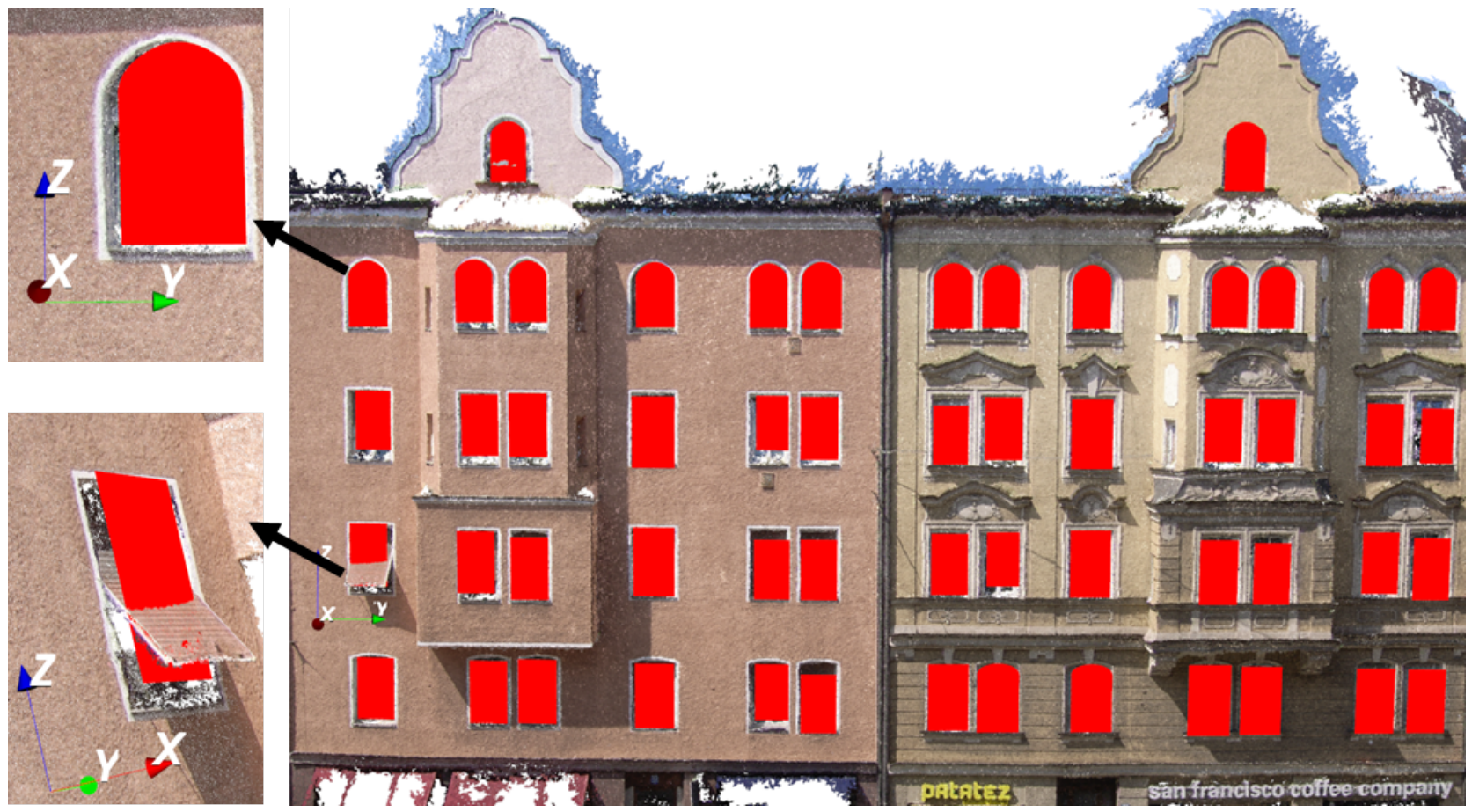

Figure 12: Filled red polygons representing localized windows and doors. Most often, segments of these outlines are embedded within the $3 D$ point clouds of the facade. As mentioned in Figure 9, dimensions of windows and doors estimates may not be the same.

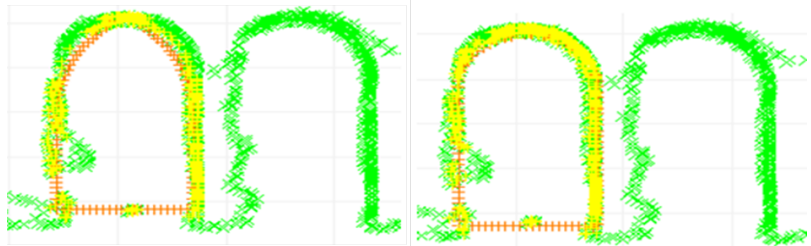

Figure 8: Inter class model selection between templates of a gothic (brown pluses on the left side) and an arched-dormer (right side) window. The inliers (yellow crosses) show a strong evidence for the arched-dormer window compared to the gothic at this position of the facade.

\section{EVALUATION}

We evaluate our algorithm based on several data sets of varying complexity characterised by point density variations, irregular and regular window and door locations, different window and door models as well as the data acquisition method (LiDAR and image matching). In each test case we downsampled the input data to a resolution of $0.03 \mathrm{~m}$ and used an MSAC inlier threshold of $0.3 \mathrm{~m}$ for building the hypothesis of the first main facade plane. For the plane-angular sweeping, a finer inlier threshold of $0.12 \mathrm{~m}$ was used and an angular deviation sampled uniformly between 0 and 3 degrees from the vertical (up) vector. We segmented five hypothesis for each facade, i.e., $\mathcal{M}=5$. Also, for every element of the compact support, we generate 30 samples using a uniform distribution, i.e., $\mathcal{N}=30$. Thus, for every search position while sliding on a facade, $5 \times 30 \times \#$ templates hypothesis are analysed. To scale the search, a 2D KD-tree is build for every boundary element using a fast approximate nearest neighbors search, e.g., (Muja and Lowe, 2009). An inlier threshold of $0.1 \mathrm{~m}$ was used within our likelihood function to score $\boldsymbol{\theta}$.

Compared to all the other MC-simulation based search methods mentioned above e.g., Dick et al. (2004), our algorithm requires a very small number of samples. We think that this is due to 


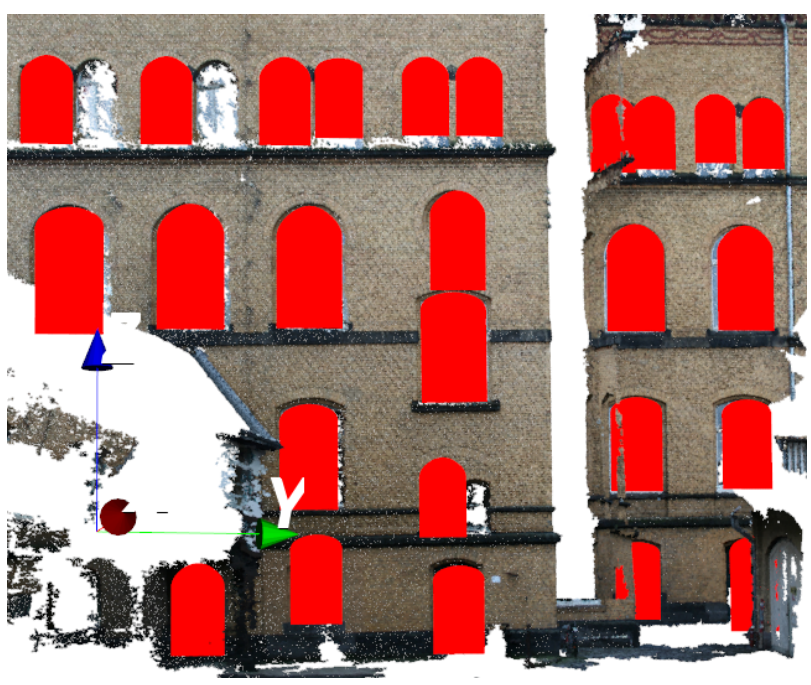

Figure 10: Two top left windows are not localized. This is because these windows are too close the neighbouring windows and their hypothesis overlaps these neighbouring windows and gets vote out due to the overlap. In general, we can remedy such problems by allowing many more samples that would increase the probability of getting a global maximum, i.e., in this case getting exactly two non-overlapping but very close windows.

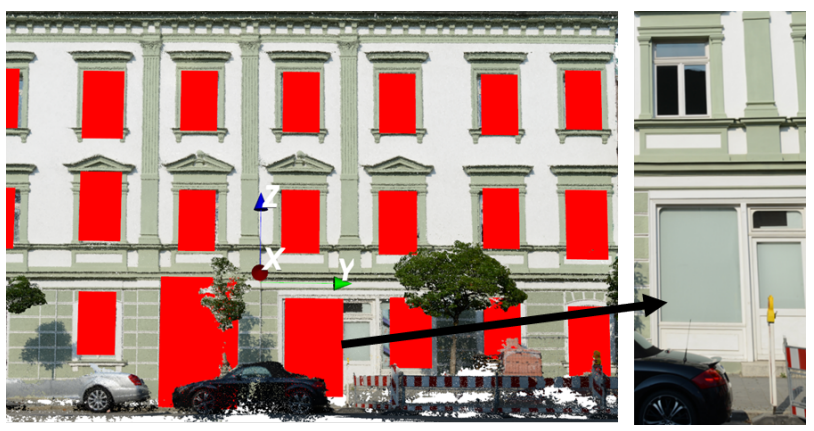

Figure 11: The left facade shows localized windows and doors (filled red polygons) of a facade. An image of a part of the facade is depicted on the right. The arrows shows a false localization of a door.

the strong hypotheses of window and door outlines generated through our combined angular-plane sweeping and boundary estimation. Our algorithm requires no parameter fine tuning for a diversity of input data compared to image based methods for finding object boundaries e.g., the Canny edge detector. However, it will show a poor performance if the 3D geometry of off-thefacade objects is lacking, e.g., for modern facades with very huge glass windows embedded within the facade, popular for office buildings.

We analyze the overall performance of our algorithm on 20 data sets: 18 from image matching e.g., (Kuhn et al., 2013) and 2 from LiDAR with a point density of $0.01 \mathrm{~m}$. For the (dense) image matching, $20-80$ images at $3264 \times 2448$ resolution were captured using a RICOH Caplio 500SE camera in a wide baseline configuration. Table 2 summarizes the results of the number of true detections of windows and doors using the templates from the span. The poor detections of the doors from the point clouds from image matching is due to the occlusion of doors by cars parked in front of the buildings. Occlusion is also a problem for the lower windows. Additionally, the poor performance on the

\begin{tabular}{lccr} 
Rectangular Template & \# objects & correct & wrong \\
\hline \#windows(image matching) & 645 & 580 & 65 \\
\#doors(image matching) & 40 & 26 & 14 \\
\#windows(LiDAR) & 35 & 34 & 1 \\
\#doors(LiDAR) & 7 & 7 & 0 \\
\hline Half-Circular Template & & & \\
\hline \#windows(image matching) & 60 & 47 & 13 \\
\#doors(image matching) & 34 & 26 & 8 \\
\#windows(LiDAR) & 12 & 12 & 0 \\
\hline Arched-Dormer Template & & & \\
\hline \#windows(image matching) & 27 & 16 & 11 \\
\hline Gothic Template & & & \\
\hline \#windows(image matching) & 15 & 13 & 2
\end{tabular}

Table 2: Results of our experiments conducted on different data sets using the four templates for windows and doors. The number of correct versus wrong localizations for 20 data sets of point clouds of facades.

detections of the arched-dormer models is due to the difficulties to distinguish between the arched-dormer and the simple rectangular template.

\subsection{Geometrical Evaluation}

We count objects as true positives if and only if the enclosed polygon defining the localised outlines is having an overlap larger than $50 \%$. We consider only rectangular windows from category Type- 1 of Table 1 for this evaluation and substantiate our choice by the frequent occurrence of this window type and the ease of annotation of bounding boxes compared to spline curves. The primary reference data set used for the evaluation concerning geometrical accuracy are self annotated bounding boxes in 3D point clouds of facades acquired by matching images. We annotated 200 bounding boxes of rectangular shaped windows from 9 facades. For each of these facades, a dominant vertical plane was segmented. All annotated bounding boxes as well as the localized rectangular window outlines are projected on the dominant plane. Next, we computed for each localized outline the intersection over union area (Jaccard Index). On this criterion, we achieved a mean accuracy of $85 \%$ for all 200 annotated bounding boxes.

\section{CONCLUSION}

We have proposed a framework for localizing windows and doors in point clouds of facades. A suitable parametrisation, likelihood and model selection combined within an MC-simulation search yields a consistent localization of facade objects (windows and doors) without any regularity assumption for the facade. Our evaluation shows the robustness and accuracy of our framework on data sets of diverse point density and origin. With the present developments in feature point detection in 3D points clouds and images, a natural extension of our approach is to incorporate a supervised learning using information gained from manually annotated input data sets. A further extension would be to evaluate our localizations for other non-rectangular shaped windows and doors with respect to accuracy using hand annotated data sets. Also, rather than using the MAP estimates, other more robust estimators such as the minimum mean squared error estimator (MMSE) could be used to improve the geometrical accuracy. 


\section{ACKNOWLEDGEMENTS}

\section{References}

Akaike, H., 1973. Information Theory and an Extension of the Maximum Likelihood Principle. In: 2nd Intern. Symp. on Information Theory, pp. 267-281.

Alegre, F. and Dellaert, F., 2004. A Probabilistic Approach to the Semantic Interpretation of Building Facades. In: International Workshop on Vision Techniques Applied, pp. 1-12.

Becker, S. and Haala, N., 2008. Integrated LIDAR and Image Processing for the Modelling of Building Facades. Photogrammetrie-Fernerkundung-Geoinformation 2008(2), pp. 65-81.

Brandenburger, W., Drauschke, M., Nguatem, W. and Mayer, H., 2013. Cornice Detection Using Facade Image and Point Cloud. Photogrammetrie-Fernerkundung-Geoinformation 2013(5), pp. 511-521.

Dick, A. R., Torr, P. H. S. and Cipolla, R., 2004. Modelling and Interpretation of Architecture from Several Images. International Journal of Computer Vision 60(2), pp. 111-134.

Ferrari, V., Tuytelaars, T. and Van Gool, L., 2006. Object detection by contour segment networks. In: Proceedings of the 9th European Conference on Computer Vision - Volume Part III, ECCV'06, Springer-Verlag, Berlin, Heidelberg, pp. 14-28.

Frahm, J.-M., Fite-Georgel, P., Gallup, D., Johnson, T., Raguram, R., Wu, C., Jen, Y.-H., Dunn, E., Clipp, B., Lazebnik, S. and Pollefeys, M., 2010. Building Rome on a Cloudless Day. In: ECCV, LNCS 6314, pp. 368-381.

Friedman, S. and Stamos, I., 2013. Online Detection of Repeated Structures in Point Clouds of Urban Scenes for Compression and Registration. International Journal of Computer Vision 102(1-3), pp. 112-128.

Fritsch, D., Becker, S. and Rothermel, M., 2013. Modeling Facade Structures Using Point Clouds From Dense Image Matching. In: International Conference on Advances in Civil, Structural and Mechanical Engineering, pp. 57-64.

Isard, M. and Blake, A., 1998. CONDENSATION: Conditional Density Propagation for Visual Tracking. International Journal of Computer Vision 29, pp. 5-28.

Kuhn, A., Hirschmueller, H. and Mayer, H., 2013. In: J. Weickert, M. Hein and B. Schiele (eds), GCPR, pp. 41-50.

Lafarge, F. and Mallet, C., 2012. Creating Large-Scale City Models from 3D-Point Clouds: A Robust Approach with Hybrid Representation. International Journal of Computer Vision 99(1), pp. 69-85.

Lin, H., Gao, J., Zhou, Y., Lu, G., Ye, M., Zhang, C., Liu, L. and Yang, R., 2013. Semantic Decomposition and Reconstruction of Residential Scenes from LiDAR Data. ACM Transactions on Graphics 32(4), pp. 66:1-66:10.

Mayer, H., Bartelsen, J., Hirschmüller, H. and Kuhn, A., 2012. Dense 3D Reconstruction from Wide Baseline Image Sets. In: Outdoor and Large-Scale Real-World Scene Analysis - 15th International Workshop on Theoretical Foundations of Computer Vision, LNCS 7474, pp. 285-304.

Muja, M. and Lowe, D. G., 2009. Fast approximate nearest neighbors with automatic algorithm configuration. In: International Conference on Computer Vision Theory and Application VISSAPP'09), INSTICC Press, pp. 331-340.
Nguatem, W., Drauschke, M. and Mayer, H., 2012. Finding cuboid-based building models in point clouds. ISPRS XXXIXB3, pp. 149-154.

Riemenschneider, H., Donoser, M. and Bischof, H., 2010. Using partial edge contour matches for efficient object category localization.

Ripperda, N. and Brenner, C., 2006. Reconstruction of Facade Structures Using a Formal Grammar and RjMCMC. In: DAGM 2006, LNCS 4174, pp. 750-759.

Rissanen, J., 1987. Minimum-Description-Length Principle. Wiley \& Sons.

Rusu, R. B., Blodow, N., Marton, Z.-C., Soos, A. and Beetz, M., 2007. Towards 3d object maps for autonomous household robots. In: Proceedings of the 20th IEEE International Conference on Intelligent Robots and Systems (IROS), San Diego, CA, USA.

Schmittwilken, J., Yang, M. Y., Förstner, W. and Plümer, L., 2009. Integration of Conditional Random Fields and Attribute Grammars for Range Data Interpretation of Man-Made Objects. Annals of GIS 15(2), pp. 117-126.

Snavely, N., Seitz, S. M. and Szeliski, R., 2006. Photo Tourism: Exploring Photo Collections in 3D. ACM Transactions on Graphics 25(3), pp. 835-846.

Torr, P. H. S. and Zisserman, A., 2000. Mlesac: A new robust estimator with application to estimating image geometry. Computer Vision and Image Understanding 78, pp. 2000.

Tuttas, S. and Stilla, U., 2013. Reconstruction of faÇades in point clouds from multi-aspect oblique als. In: ISPRS Annals of the Photogrammetry, Remote Sensing and Spatial Information Sciences, Volume II-3/W3, 2013 CMRT13 - City Models, Roads and Traffic 2013. 